\title{
Rescue operations on dedicated high speed railway lines
}

\author{
R. Takagi \\ Kogakuin University, Japan
}

\begin{abstract}
When disruptions of service take place on dedicated high speed railway lines, it is not uncommon that situations arise in which special "rescue" operations would be necessary. This paper outlines the following; 1) how such situations take place, or how efforts are being made to avoid them; 2) how rescue operations can be done; and 3) possible research and development on how the situations can be reduced using new technologies.
\end{abstract}

Keywords: rescheduling, rescue operations, high speed railways, substitute train protection, on-board energy storage.

\section{Introduction}

When disruptions of service take place on dedicated high speed railway lines, trains may have to be halted at places where passengers on board the trains cannot evacuate. For example, it has been reported on the Asahi Shimbun [1] that, on 29 January 2010, five trains with approximately 3,100 passengers on board had been stranded for nearly four hours on the Tōkaidō Shinkansen in Japan after a power outage caused by the breakage of an auxiliary messenger wire of the compound overhead line equipment. Earlier, it has been reported on the BBC News Website [2] that five Eurostar trains got stuck inside the Channel Tunnel when exceptional weather conditions caused failures of electrical systems on board trains, with nearly 2,000 passengers having to be rescued in a series of special operations.

In this paper, the following will be outlined: 1) how such situations take place, or how efforts are being made to avoid them; 2) how rescue operations are currently carried out and can be done; and 3) possible research and development on how the situations can be reduced using new technologies. 


\section{The need for rescue operations}

There may be situations in which high speed trains stop at places other than stations or emergency evacuation points along a dedicated high speed railway line when a disruption to services take place. If this situation is expected to continue for an unacceptably long time, rescue operations must take place to let these passengers out of the trains.

Once the disruption takes place, and the information on the nature and the extent of the incident that caused the disruption is available, traffic control is done to avoid such out-of-station halts. However, it is difficult to avoid such situations before the information is available, especially when the average distance between stations and/or evacuation points is long and the frequency of the trains is high.

\section{How rescue operations are being done}

If a train to be rescued can be moved, but something is blocking its way, the first thing to be done is to open a path for the train so that it can be moved to a nearest station or an evacuation point. It will include moving the train in the direction different from the one it was originally travelling towards. This, however, may need bi-directional signalling, which is uncommon for the Japanese high speed railway system, Shinkansen, and is not effective for high speed railways like Shinkansen where train frequency is very high. If the distance for which the train must do the reverse-running is short, the train may actually do so in the rescue operation.

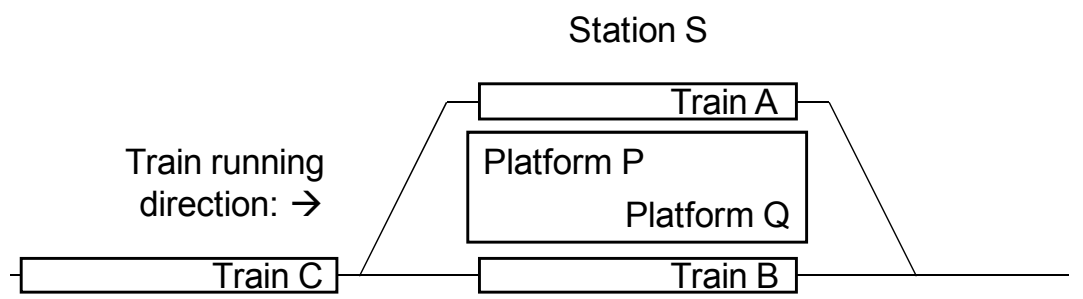

Figure 1: An example rescue operation at a station (1).

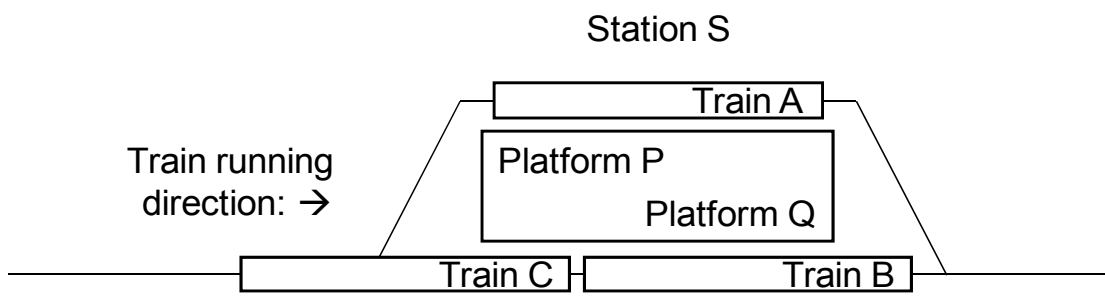

Figure 2: An example rescue operation at a station (2). 
If the train cannot be moved by itself, but can be pulled to the nearest station or rescue point if another train or a rescue locomotive is attached to it, this option will be tried.

Finally, if the train cannot be moved for various reasons, but another road in a double-track railway is open, a rescue train is prepared and sent to the site where the train is halted. The passengers on board the unmovable train will be transferred to the rescue train at the site and transported to the nearest station or evacuation point.

Sometimes it may be necessary to put more than one train on a platform which normally serves only one. An example is shown in Figures 1 and 2. In Figure 1, there are three trains A, B and C, out of which Train C is not on any of the platforms of Station S. By moving Train B slightly forward, a part of Train C can share Platform Q with Train B, and the passengers on Train C can safely alight using the passenger doors towards the front of it.

\section{Discussions on how to improve rescue operations, or how to avoid this happening on a massive scale}

\subsection{Information available to the line controllers}

If a disruptive event takes place in a railway line which may lead to the requirement for any rescue operations as discussed above, it is always important that the line controllers have information as much and as accurate as possible on the nature and the extent of the event.

Especially important is the accurate estimate as to how long it will take to remove the cause of the blockage, which will have an impact on the optimal overall re-scheduling strategy. The rescue operation itself may take time, and it may be better to just wait until the cause of the disruption is removed if the estimated time until the cause is removed is short enough.

Unfortunately, currently this estimate is very inaccurate, which causes the decision to start the rescue operation to be very late. The development of technologies to improve the precision of the estimate, including construction of a good incident database and good information acquisition at the control room, is strongly expected.

\subsection{Substitute train protection}

Generally speaking, a high speed railway line is equipped with a train protection system throughout its length, which is in full use during normal operations. However, during rescue operations, substitute train protection systems are frequently used, with special arrangements and restrictions being imposed.

For example, for safety reasons the following rules are imposed when a substitute train protection is being used on a Japanese Shinkansen line, as explained in [3]: 1) every train must have a driver and a guard (or two drivers) on the leading driver's cab;2) train speed is limited to $110 \mathrm{~km} / \mathrm{h} ; 3$ ) every train must stop before entering a station; and 4) every train must stop at all stations. 
A substitute train protection is basically a fully manual process, which is prone to human errors. Okada [4] introduced the intention of JR East to develop a new substitute train protection system using the digital train radio, which is expected to be less prone to errors.

\subsection{Energy storage onboard trains}

Energy storage systems are expected to reduce energy consumption of electric railways, especially DC railways. The application of energy storage systems to high speed railway systems will not, however, contribute to any considerable energy-savings; this is because high speed railways are generally AC-fed.

Nevertheless, it is expected that the application of energy storage on-board high speed railcars will contribute to the improvement in the rescue operations, mainly because this may make it possible to move trains regardless of the availability of electric power through overhead contact equipment.

Disruption of power supply of a railway line will result in major disruption of rail services on the line, because the loss of power means loss of the ability to move for the trains in a certain area. If some amount of energy is stored onboard, the ability to move will not be lost entirely even when the failure of power supply takes place; this will mean it is much easier to plan and carry out rescue operations under such circumstances.

Careful design of the railcars, however, must be made if this idea is to be implemented. The addition of on-board energy storage will result in increased weight, and therefore increased energy consumption and wear and tear of the tracks. This must be compared to the advantage gained by the addition.

\section{Conclusion}

Rescue operations are the necessary step in the train re-scheduling when a major disruption of service takes place. As discussed in Section 4, the development of good system to give precision estimate of the time to the removal of the blocking condition is very important. In addition, there are some new technologies that may contribute to the improved rescue operations, especially on-board energy storage.

The rescue operation, however, is only necessary when the major disruption actually happens. In this respect, the improvement of the reliability of services, by improving the reliability of individual components that make up the whole railway system, is most important.

\section{References}

[1] Asahi Shimbun, 30 January 2010 (in Japanese).

[2] BBC News Website, 19 December 2009.

[3] "Shinkansen Signalling Installations" (in Japanese), Revised Ed., Railway Electrical Engineers' Association of Japan (2002). 
[4] Okada, K.: "Development and Implementation of Digital ATC Systems" (in Japanese), JR-East Technical Review, 5, pp. (2003). http://www.jreast.co.jp /development/tech/pdf_5/27-30.pdf (accessed 1 May 2010). 